\title{
Interpolation and partial differential equations
}

\author{
Lech Maligranda \\ Department of Mathematics, Luleå University of Technology, S-95187 Luleå, Sweden \\ Lars Erik Persson \\ Department of Mathematics, Luleå University of Technology, S-95187 Luleå, Sweden \\ and Department of Mathematics, Narvik Institute of Technology, P.O. Box 385, \\ N-8501 Narvik, Norway \\ John Wyller \\ Department of Mathematics, Narvik University of Technology, P.O. Box 385, \\ N-8501 Narvik, Norway
}

(Received 31 March 1994; accepted for publication 8 April 1994)

One of the main motivations for developing the theory of interpolation was to apply it to the theory of partial differential equations (PDEs). Nowadays interpolation theory has been developed in an almost unbelievable way ssee the bibliography of Maligranda [Interpolation of Operators and Applications (1926-1990), 2nd ed. (Luleå University, Luleå, 1993), p. 154]\}. In this article some model examples are presented which display how powerful this theory is when dealing with PDEs. One main aim is to point out when it suffices to use classical interpolation theory and also to give concrete examples of situations when nonlinear interpolation theory has to be applied. Some historical remarks are also included and the relations to similar results are pointed out.

\section{INTRODUCTION}

One of the main motivations for developing the theory of interpolation was to apply it to the theory of partial differential equations (PDEs). The original definitions in real interpolation theory worked out by Gagliardo, Lions, Peetre, etc. were usually given in terms of PDEs (the trace theorem). However, around 1960 it was discovered (by Peetre) that these interpolation spaces equivalently could be described (via the parameter theorem) by using the so-called $K$ functional (see our Sec. II). From the 1960s until today the interpolation theory has been developed in an unbelievable way as an art in its own right (with separate conferences with hundreds of participants, etc.). See, e.g., the books by Bergh-Löfström, ${ }^{1}$ Triebel, ${ }^{2}$ Krein-Petunin-Semenov, ${ }^{3}$ and especially the bibiliography of Maligranda. ${ }^{4}$ However, in our opinion, many genuine new applications of this powerful theory have not yet been pointed out, and many times the original arguments for developing the theory have been forgotten or not emphasized.

In the present article we will point out the fact that the present state of the interpolation theory is a very powerful tool for obtaining qualitative information from the boundary data about the solutions of PDEs. We start out by employing classical linear interpolation theory to the linear wave equation. However, in many cases when studying nonlinear problems, we need powerful tools of modern nonlinear interpolation theory. We briefly discuss the latter theory and prove some new regularity results for the $p$-Laplace and the Korteweg-de Vries equations. Finally, we compare our results with other similar results and shortly discuss the possibilities of further developing and applying these ideas.

This article is organized as follows: In Sec. II we give a preliminary discussion on interpolation theory and PDEs including historical remarks, a description of (Lions-Peetre's) real interpolation theory, and a brief description of the main ideas of this article. In Sec. III we prove and discuss an application of linear interpolation theory to a wave equation with variable coefficients (Theorem 1). In Sec. IV we present and discuss a useful interpolation theorem for nonlinear operators (Theorem 1). Section $\mathrm{V}$ is devoted to the discussion of the $p$-Laplace equation and we 
point out that the physical information inherited in the boundary data can be carried over to the corresponding solutions by using the nonlinear interpolation theory (see Theorems 1 and 2). In Sec. VI we do a similar investigation concerning the Korteweg-de Vries equation (see Theorem 1). Finally, Sec. VII is reserved for a final discussion and some concluding remarks, and some open questions which we hope are of interest for people working in both the fields of physics and pure mathematics and which probably must be tackled by using ideas from both these disciplines.

\section{A PRELIMINARY DISCUSSION ON INTERPOLATION THEORY AND PDES}

\section{A. The state of the art}

The first interpolation theorem (the Riesz-Thorin interpolation theorem, see Ref. 1 p. 2 and Ref. 5) was proven by Riesz already in 1926. This theorem was the first step in creating the (Calderón) complex method of interpolation [.] $]_{\theta}$. In a similar way, the Marcinkiewicz interpolation theorem from 1939 (see Ref. 1, p. 9 and Ref. G) inspired the (Lions-Peeue) real method of interpolation $(.)_{\theta, q}$. Nowadays, there exist several methods of interpolation, but the real and complex methods are sufficient for most applications of linear problems. Furthermore, the interpolation spaces at hand are described explicitely in almost all situations of practical importance (see, e.g., Refs. 1-4) and, thus, the results can be given in familiar terms.

\section{B. On (Lions-Peetre) real method of interpolation}

For the purposes of this article, it is sufficient to work with the real method, and in what follows we only describe this method. The starting point is to consider a Banach pair $\bar{A}=\left(A_{0}, A_{1}\right)$ consisting of two Banach spaces $A_{0}$ and $A_{1}$, which both are continuously embedded in a (large) Haussdorff topological vector space. For $0<\theta<1$ and $1 \leqslant q \leqslant \infty$, the real interpolation space $\bar{A}_{\theta, q}=\left(A_{0}, A_{1}\right)_{\theta, q}$ consists of all $a \in \Lambda_{0}+\Lambda_{1}$ satisfying

$$
\|a\|_{\bar{A}_{\theta, q}} \equiv\|a\|_{\theta, q}=\left\{\begin{array}{l}
\left(\int_{0}^{\infty}\left(t^{-\theta} K(t, a)\right)^{q} \frac{d t}{t}\right)^{1 / q}, \quad q<\infty \\
\sup _{t>0}\left(t^{-\theta} K(t, a)\right), \quad q=\infty,
\end{array}\right.
$$

where $K(t, a) \equiv K\left(t, a, A_{0}, A_{1}\right)$ is the Peetre $K$ functional defined by

$$
K(t, a) \equiv \inf \left\{\left\|a_{0}\right\|_{A_{0}}+t\left\|a_{1}\right\|_{A_{1}}: a=a_{0}+a_{1}, a_{0} \in A_{0}, a_{1} \in A_{1}\right\}
$$

It is easy to see that $A_{\theta, q}$ is an intermediate space with respect to the Banach couple $\bar{A}$, i.e., that $A_{0} \cap A_{1} \subset A \subset A_{0}+A_{1}$ and $A_{\theta, q}$ is an interpolation construction in the following sense: For any linear operator $T: A_{0}+A_{1} \rightarrow B_{0}+B_{1}$ which maps $A_{0}$ boundedly into $B_{0}$ with norm $M_{0}$ and $A_{1}$ boundedly into $B_{1}$ with norm $M_{1}, T$ maps $\bar{A}_{\theta, q}$ boundedly into $\bar{B}_{\theta, q}$ with the norm less than $M_{0}^{1-\theta} M_{1}^{\theta}$.

If $A_{1} \subset A_{0}$, then it is easy to see that

$$
A_{1} \subset A_{\theta_{0}, q_{0}} \subset A_{\theta_{1}, q_{1}} \subset A_{0}
$$

provided that $\theta_{0}>\theta_{1}$ or that $\theta_{0}=\theta_{1}$ and $q_{0} \leqslant q_{1}$. Here we use the convention that $A_{0, q}=A_{0}$ and $A_{1, q} \equiv A_{1}$.

For more details and proofs concerning this interpolation theory of linear operators, we refer to the original article ${ }^{7}$ of Peetre and the books in Refs. 1-3. Moreover, concerning the definitions of the function spaces we are working with in this article, we refer to the books in Refs. 1-3 and the classical book ${ }^{8}$ by Adams. 


\section{The main idea}

Let $P(x, t), x \in \Omega \subset \mathbf{R}^{n}, t \in \mathbf{R}$ be a partial differential operator and consider the PDE

$$
\left\{\begin{array}{l}
P u=g \\
u=f
\end{array}\right.
$$

and the family of operators $\left\{T_{t}\right\}, 0 \leqslant t<\infty$, where $T_{t}$ maps the boundary value function $f(x)$ into the solution $u(x, t)$ of $(2.1)$, i.e.,

$$
T_{t} f(x)=u(x, t)
$$

Now, if these operators have the boundedness properties

$$
\left\|T_{t} f\right\|_{B_{0}} \leqslant C_{0}\|f\|_{A_{0}}, \quad\left\|T_{t} f\right\|_{B_{1}} \leqslant C_{1}\|f\|_{A_{1}}
$$

for all $f \in A_{0} \cap A_{1}$, where $\left(A_{0}, A_{1}\right)$ and $\left(B_{0}, B_{1}\right)$ are Banach pairs, then, by interpolation, we can control the boundedness in all "intermediate" cases, i.e.,

$$
\left\|T_{t} f\right\|_{\left(B_{0}, B_{1}\right)_{\theta, q}} \leqslant C_{0}^{1-\theta} C_{1}^{\theta}\|f\|_{\left(A_{0}, A_{1}\right)_{\theta, q}}
$$

for all $f \in A_{0} \cap A_{1}$. Moreover, if we have some additional information about the operators $T_{t}$ (e.g., that they are Lipschitz, monotone, etc.), then we can extend the inequality (2.3) to hold even on the closure of $A_{0} \cap A_{1}$ in $\left(A_{0}, A_{1}\right)_{\theta, q}$ [when $q<\infty$ this closure in fact coincides with $\left(A_{0}, A_{1}\right)_{\theta, q}$.

However, if we have the explicit additional information that $A_{1} \subset A_{0}$, then the assumptions in Eq. (2.2) can be replaced by

$$
\begin{gathered}
\left\|T_{t} f\right\|_{B_{0}} \leqslant C_{0}\|f\|_{A_{0}}, \quad f \in A_{0}, \\
\left\|T_{t} f\right\|_{B_{1}} \leqslant C_{1}\|f\|_{A_{1}}, \quad f \in A_{1}
\end{gathered}
$$

and the conclusion is that the inequality in (2.3) holds for every $f$ from the spaces $\left(A_{0}, A_{1}\right)_{\theta, q}$.

Nowadays, we have concrete descriptions of the spaces $\left(A_{0}, A_{1}\right)_{\theta, q}$ in most cases of practical importance. Therefore, this technique is very powerful and easy to use.

\section{A concrete example}

Assume that $1 \leqslant p_{0}, p_{1}, q_{0}, q_{1} \leqslant \infty$, and

$$
\left\|T_{t} f\right\|_{L_{q_{0}}} \leqslant A t^{\alpha}\|f\|_{L_{\rho_{0}}}
$$

and

$$
\left\|T_{t} f\right\|_{L_{q_{1}}} \leqslant B t^{\beta}\|f\|_{L_{p_{1}}}
$$

It is well known that

$$
\left(L_{p_{0}}, L_{p_{1}}\right)_{\theta, p_{\theta}}=L_{p_{\theta}}, \quad \frac{1-\theta}{p_{0}}+\frac{\theta}{p_{1}}=\frac{1}{p_{\theta}}
$$

and, thus, by using real interpolation, we find that

$$
\left\|T_{t} f\right\|_{L_{q_{\theta}}} \leqslant C t^{\alpha(1-\theta)+\beta \theta}\|f\|_{L_{p_{\theta}}},
$$


where

$$
\frac{1}{q_{\theta}}=\frac{1-\theta}{q_{0}}+\frac{\theta}{q_{1}}, \quad \frac{1}{p_{\theta}}=\frac{1-\theta}{p_{0}}+\frac{\theta}{p_{1}}, \quad 0 \leqslant \theta \leqslant 1 .
$$

Note that $p_{\theta}\left(q_{\theta}\right)$ are real numbers between $p_{0}$ and $p_{1}\left(q_{0}\right.$ and $\left.q_{1}\right)$.

Remark: In many applications one of the estimates (2.5) and (2.6) can be taken as an energy estimate (e.g., $p_{0}=q_{0}=2$ and $\alpha=1$; cf. Theorem 1).

\section{E. On nonlinear PDEs and the interpolation theory}

We pronounce that several important physical phenomena can be modeled by means of nonlinear PDEs. Such equations can be formulated in terms of nonlinear differential operators. Fortunately, nowadays we know fairly well that several operators of this type can be interpolated in a similar way as above. In practice the information in the extremal cases is usually known as norm estimates (of the "energy" type) and generalized variants of Hölder, (semi-)Lipschitz, or sublinear conditions, respectivcly. Nowadays we have fairly good knowledge about interpolation results in such cases (see the review article in Ref. 9 and the references given there). This fact is of outstanding importance for several applications and is one of the main objectives for writing the present article (see, e.g., Secs. 5 and 6).

\section{AN APPLICATION OF LINEAR INTERPOLATION THEORY TO A WAVE EQUATION WITH VARIABLE COEFFICIENTS}

Consider the following Cauchy problem for the one-dimensional wave equation

$$
\left.\begin{array}{c}
u_{t t}(x, t)=\left(a(x) u_{x}(x, t)\right)_{x}, \quad x \in \mathbf{R}, \quad t>\mathbf{0}, \\
\left.u\right|_{t=0}=0, \quad x \in \mathbf{R}, \\
\left.u_{t}\right|_{t=0}=f(x), \quad x \in \mathbf{R} .
\end{array}\right\}
$$

Let $u$ denote the solution of Eq. (3.1), and let $T_{t}$ be the evolution operator defined by $T_{t} f(x) \equiv u(x, t)$. find that

Case 1: $a(x)$ is constant $[a(x)=c]$. Then, by using Young's inequality for convolution we

$$
\|u(\cdot, t)\|_{p}=\left\|T_{t} f\right\|_{p} \leqslant C t\|f\|_{p} .
$$

Case 2: Assume that $a(x)$ is essentially bounded, i.e., that

$$
0<c \leqslant a(x) \leqslant c<\infty .
$$

The main goal of this section is to present a simple (interpolation) proof of the fact that Eq. (3.2) also holds in this case.

Theorem 1: If $a(x)$ satisfies Eq. (3.3) and $2 \leqslant p \leqslant \infty$, then the inequality (3.2) holds true.

Proof: For $p=2$, we have the "energy" estimate

$$
\|u(\cdot, t)\|_{2}=\left\|T_{t} f\right\|_{2} \leqslant t\|f\|_{2}, \quad \forall f \in L^{2} .
$$

If $f \in L^{\infty}$, then the Hölder inequality yields

$$
\|u(\cdot, t)\|_{\infty}=\left\|T_{L} f\right\|_{\infty} \leqslant 4 \sqrt{\frac{A}{a}} t\|f\|_{\infty} .
$$


Hence the operators $T_{t}: L^{2} \rightarrow L^{2}$ and $T_{t}: L^{\infty} \rightarrow L^{\infty}$ are bounded. Then, by applying RieszThorin's theorem with $p_{0}=q_{0}=2, p_{1}=q_{1}=\infty$, and $\theta=(p-2) / p$, we find that $T_{t}$ is a bounded operator from $L^{p}$ into $L^{p}, 2<p<\infty$, such that

$$
\|u(\cdot, t)\|_{p}=\left\|T_{t} f\right\|_{p} \leqslant\left(4 \sqrt{\frac{A}{a}} t\right)^{1-\theta} t^{\theta}\|f\|_{p}=\left(4 \sqrt{\frac{A}{a}}\right)^{2 / p} t\|f\|_{p}, \quad \forall f \in L^{p} .
$$

Remark: A characteristic feature for the $n$-dimensional case $(n>1)$ is that the estimate (3.2) is true only for $p$ in the neighborhood of $p=2$ even if $a(x)=c$ (Case 1 ).

\section{A USEFUL INTERPOLATION RESULT FOR LOCALLY HÖLDER OPERATORS}

Many nonlinear differential operators of practical importance may be regarded as some type of locally Hölder operators (see, e.g., our examples given in Secs. V and VI). In this section we will present and discuss an interpolation theorem of Maligranda ${ }^{10}$ which deals with very general operators of this kind. First we need the following definition:

Definition 1: Let $0<\theta<1$ and $0<p \leqslant \infty$. We say that the pair $\left(A_{0}, A_{1}\right)$ has a $(\theta, p)$ approximate identity if there is a family of continuous mappings $S_{t}: A_{\theta, p} \rightarrow A_{1}$ for $0<t \leqslant 1$, such that

$$
1^{0}\left\|S_{t} a\right\|_{\theta, p}+t^{-\theta}\left\|S_{t} a\right\|_{A_{1}} \leqslant C\|a\|_{\theta, p}, \quad \text { for all } a \in A_{\theta, p} \text { and } t \in[0,1]
$$

and

$$
2^{0}\left\|S_{t} a-a\right\|_{o, p}+t^{-\theta}\left\|S_{t} a-a\right\|_{A_{0}} \rightarrow 0 \quad a s \quad t \downarrow 0 \text { for } a \in A_{\theta, p}
$$

and it is uniform on compact subsets of $A_{\theta, p}$.

Example 1 (Bona and Scott $\left.{ }^{\prime \prime}\right)$ : The pair $\left(L^{2}(\mathbf{R}), H^{m}(\mathbf{R})\right)$, where $m$ is a positive integer, has a $(\theta, 2)$ approximate identity.

Theorem 1: (Maligranda ${ }^{10}$, cf. also Ref. 12) Let $A_{1} \subset A_{0}, B_{1} \subset B_{0}$, and $0 \leqslant \mu<1,0<r, \alpha_{0}$, $\alpha_{1} \leqslant \infty$. Suppose that $T$ is a mapping such that

(i) $T: A_{\mu, r} \rightarrow B_{0}$ and, for $a, b \in A_{\mu, r}$

$$
\|T a-T b\|_{B_{0}} \leqslant \varphi\left(\|a\|_{\mu, r},\|b\|_{\mu, r},\|a-b\|_{\mu, r}\right)\|a-b\|_{A_{0}}^{\alpha_{0}}
$$

and

(ii) $T: A_{l} \rightarrow B_{I}$ and. for $a \in A_{I}$

$$
\|T a\|_{B_{1}} \leqslant \psi\left(\|a\|_{\mu, r}\right)\|a\|_{A_{1}}^{\alpha_{1}},
$$

where $\varphi: \mathbf{R}_{+}^{3} \rightarrow \mathbf{R}$ is continuous and nondecreasing in each variable and $\psi \cdot \mathbf{R}_{+} \rightarrow \mathbf{R}_{+}$is continuous and nondecreasing. If $\theta>\mu$ or $\theta=\mu$ and $p \leq r$, then $T$ maps $A_{\theta, p}$ into $B_{\eta, q}$ and, for $a \in A_{\theta, p}$,

$$
\|T a\|_{\eta, q} \leqslant C h\left(\|a\|_{\mu, r}\right)\|a\|_{\theta, p}^{\alpha}
$$

where

$$
\eta=\theta \frac{\alpha}{\alpha_{1}}, \quad \alpha=(1-\eta) \alpha_{0}+\eta \alpha_{1}, \quad p=\alpha q, \quad h(t)=\varphi(t, 2 t, 2 t)^{1-\eta} \psi /(2 t)^{\eta}
$$

In addition, assume that the pair $\left(A_{0}, A_{l}\right)$ has a $(\theta, p)$ approximate identity $\left\{S_{t}\right\}$ for some $\theta>\mu$ or $\theta=\mu$ and $p \leqslant r$, and that 
(iii) $T$ is continuous as a map of $A_{1}$ to $B_{l}$.

Then $T$ is a continuous map from $A_{\theta, p}$ to $B_{\eta, q}$.

Remark 1: The above theorem was previously obtained in the following special cases:

(a) $\mu=0, \varphi=\psi=$ const, and $\alpha_{0}=\alpha_{1}=\alpha=1$ [Lions $^{13-15}$ (Theorem 3.1) and Peetre ${ }^{16}$ (Theorem 3.1)],

(b) $\quad \mu=0$ and $\varphi(u, v, w)=C(u, v)\left[\operatorname{Tartar}^{17}\right.$ (Theorem 2)],

(c) $\varphi(u, v, w)=C(u+v)$ and $\alpha_{0}=\alpha_{1}=1$ [Bona and $\mathrm{Scott}^{11}$ (Theorem 1)].

In the next two sections we will demonstrate how Theorem 1 can be used to obtain regularity information about the solutions of the $p$-Laplace equation and the Korteweg-de Vries equation.

\section{A REGULARITY RESULT FOR THE $p$-LAPLACIAN EQUATION}

For $1<p<\infty$, let

$$
W^{1, p}=\left\{u \in L^{p}\left(\mathbf{R}_{+}^{n}\right): \frac{\partial u}{\partial x_{i}} \in L^{p}\left(\mathbf{R}_{+}^{n}\right), i=1,2, \ldots, n\right\},
$$

with the norm

$$
\|u\|_{W^{1, p}}=\left(\|u\|_{p}^{p}+\sum_{i=1}^{n}\left\|\frac{\partial u}{\partial x_{i}}\right\|_{p}^{p}\right)^{1 / p} .
$$

Moreover, let $W_{0}^{1, p}$ denote the closure of $\mathscr{C}$ in $W^{1, p}$ and $W^{-1, p^{\prime}}$ the dual space of $W_{0}^{1, p}$. Then the operator $T: W_{0}^{1, p} \rightarrow W^{-1, p^{\prime}}$, as a dual mapping on $W_{0}^{1, p}$, defined by the formula

$$
T u \equiv-\sum_{i=1}^{n} \frac{\partial}{\partial x_{i}}\left(\left|\frac{\partial u}{\partial x_{i}}\right|^{p-2} \frac{\partial u}{\partial x_{i}}\right)+|u|^{p-2} u
$$

is strictly monotone, $h$ continuous and coercive, and therefore, by the Browder-Minty theorem, for every $f \in W^{-1, p^{\prime}}$ there is a unique $u \in W_{0}^{1, p}$ such that

$$
T u=f .
$$

Our regularity result reads:

Theorem 1: Let $2 \leqslant p<\infty$. If $f \in\left(W^{-1, p^{\prime}}, L^{2} \cap W^{-l, p^{\prime}}\right)_{\theta, q}$ then the solution $u$ of the equation $T u=f$ belongs to $\left(W_{0}^{l, p}, L^{2(p-l)}\right)_{\theta, q}$ and $\|u\|_{\theta, q} \leqslant C\|f\|_{\theta, q}^{1 / p-1}$.

Proof: The first step consists of proving that the inverse mapping $T^{-1}: W^{-1, p^{\prime}} \rightarrow W_{0}^{1, p}$, is a Hölder mapping of order $\alpha=1 /(p-1)$. Since $p \geqslant 2$, we have

$$
\left(|a|^{p-2} a-|b|^{p-2} b\right)(a-b) \geqslant 2^{2-p}|a-b|^{p}, \quad \forall a, b \in \mathbf{R} .
$$

Hence, by putting $a=u, b=v$ and $a=\partial u / \partial x_{i}, b=\partial v / \partial x_{i}$, and integrating, one obtains

$$
(T u-T v, u-v) \equiv \int(T u-T v)(u-v) d x \geqslant 2^{2-p}\|u-v\|^{p}, \quad \forall u, v \in W_{0}^{1, p} .
$$

Therefore, by the Hölder inequality

$$
2^{2-p}\|u-v\|^{p} \leqslant(T u-T v, u-v) \leqslant\|u-v\|\|T u-T v\|_{*}
$$

and, thus 


$$
\left\|T^{-1} f-T^{-1} g\right\|^{p-1} \leqslant 2^{p-2}\|f-g\|_{*}
$$

or, equivalently

$$
\left\|T^{-1} f-T^{-1} g\right\| \leqslant 2^{(p-2) /(p-1)}\|f-g\|_{*}^{1 /(p-1)}, \quad \forall f, g \in W^{-1, p^{\prime}} .
$$

Second, we have

$$
\left\|T^{-1} f\right\|_{L^{2(p-1)}} \leqslant\|f\|_{L^{2}}^{1 /(p-1)}, \quad \forall f \in L^{2} \cap W^{-1, p^{\prime}} .
$$

We prove this fact in the following way: For every $\varepsilon>0$, let $u_{\varepsilon}$ be a solution of the equation

$$
u_{\varepsilon}+\varepsilon\left|u_{\varepsilon}\right|^{p-2} u_{\varepsilon}=u
$$

Then $\left(\partial u_{\varepsilon} / \partial x_{i}\right)\left[1+(p-1) \varepsilon\left|u_{\varepsilon}\right|^{p-2}\right]=\partial u / \partial x_{i}$, and $u_{\varepsilon}, u_{\varepsilon}\left|u_{\varepsilon}\right|^{p-2} \in W_{0}^{1, p}$. Since

$$
\begin{aligned}
\int-\frac{\partial}{\partial x_{i}}\left(\left|\frac{\partial u}{\partial x_{i}}\right|^{p-2} \frac{\partial u}{\partial x_{i}}\right)\left|u_{\varepsilon}\right|^{p-2} u_{\varepsilon} d x & =\int\left|\frac{\partial u}{\partial x_{i}}\right|^{p-2} \frac{\partial u}{\partial x_{i}} \frac{\partial}{\partial x_{i}}\left(\left|u_{\varepsilon}\right|^{p-2} u_{\varepsilon}\right) d x \\
& =(p-1) \int\left|\frac{\partial u}{\partial x_{i}}\right|^{p-2} \frac{\partial u}{\partial x_{i}} \frac{\partial u_{\varepsilon}}{\partial x_{i}}\left|u_{\varepsilon}\right|^{p-2} d x \geqslant 0
\end{aligned}
$$

and

$$
u_{\varepsilon} \leqslant u_{\varepsilon}\left[1+\varepsilon\left|u_{\varepsilon}\right|^{p-2}\right]=u
$$

it follows that

$$
\begin{aligned}
\left\|u_{s}\right\|_{L^{2(p-1)}}^{2(p-1)} & =\int\left|u_{\varepsilon}\right|^{2(p-1)} d x=\int\left|u_{\varepsilon}\right|^{p-2} u_{\varepsilon}\left|u_{\varepsilon}\right|^{p-2} u_{\varepsilon} d x \\
& \leqslant \int|u|^{p-2} u\left|u_{\varepsilon}\right|^{p-2} u_{\varepsilon} d x \\
& \leqslant \int|u|^{p-2} u\left|u_{\varepsilon}\right|^{p-2} u_{\varepsilon} d x+\sum_{i=1}^{n} \int-\frac{\partial}{\partial x_{i}}\left(\left|\frac{\partial u}{\partial x_{i}}\right|^{p-2} \frac{\partial u}{\partial x_{i}}\right)\left|u_{\varepsilon}\right|^{p-2} u_{\varepsilon} d x \\
& =\int T u \cdot\left|u_{\varepsilon}\right|^{p-2} u_{\varepsilon} d x
\end{aligned}
$$

Then, by using Schwarz inequality, we get

$$
\left\|u_{\varepsilon}\right\|_{L^{2(p-1)}}^{2(p-1)} \leqslant\|T u\|_{L^{2}}\left\|\left.u_{\varepsilon}\right|^{p-2} u_{\varepsilon}\right\|_{L^{2}}=\|T u\|_{L^{2}}\left\|u_{\varepsilon}\right\|_{L^{2(p-1)}}^{p^{-1}}
$$

or

$$
\left\|u_{e}\right\|_{L^{2(p-1)}}^{p-1} \leqslant\|T u\|_{L^{2}}=\|f\|_{L^{2}}
$$

which means that the sequence $\left\{u_{\varepsilon}\right\}$ is bounded in $L^{2(p-1)}$. Moreover, since

$$
\left\|u-u_{\varepsilon}\right\|_{L^{2}}=\varepsilon\left\|\left.u_{\varepsilon}\right|^{p-2} u_{\varepsilon}\right\|_{L^{2}}=\varepsilon\left\|u_{\varepsilon}\right\|_{L^{2(p-1)}}^{p-1} \rightarrow 0
$$

as $\varepsilon \rightarrow 0$, we have that $u \in L^{2(p-1)}$ and 


$$
\|u\|_{L^{2(p-1)}}^{p-1} \leqslant \liminf _{\varepsilon \rightarrow 0}\left\|u_{\varepsilon}\right\|_{L^{2(p-1)}}^{p-1} \leqslant\|T u\|_{L^{2}}=\|f\|_{L^{2}}
$$

which is Eq. (5.3). Finally, by using Theorem 1 with $\alpha_{0}=\alpha_{1}=1 /(p-1)$, we obtain

$$
\|u\|_{\theta, q} \leqslant 2(p-1)^{-1 / q} 2^{(p-2)(1-\theta)}\|f\|_{\theta, q}^{1 /(p-1)}
$$

and the proof is complete.

We close this section by stating the following complement to Theorem 1 :

Theorem 2: Let $l<p \leqslant 2$. If $f \in\left(W^{-1, p^{\prime}}, L^{2} \cap W^{-1, p^{\prime}}\right)_{\theta, q}$, then the solution of the equation $T u=f$ belongs to $\left(W_{0}^{l, p}, L^{2} \cap W^{-1, p^{\prime}}\right)_{\eta, \tau}$ where $\eta=\theta q(p-I) / \tau$ and $\tau=\max (1, q(p-1) /[\eta(2-p)+p-1])$.

Proof (sketch): It can be proven that

$$
\left\|T^{-1} f-T^{-1} g\right\| \leqslant c_{p}\left(\|f\|_{*}^{1 /(p-1)}+\|g\|_{*}^{1 /(p-1)}\right)^{2-p}\|f-g\|_{*}
$$

and then, by using Theorem 1 with $\alpha_{0}=1$ and $\alpha_{1}=1 /(p-1)$ and inequality (5.4), we get the result with the estimate

$$
\|u\|_{\eta, \tau} \leqslant C\left\|_{f}\right\|_{*}^{(2-p) /(p-1)}\|f\|_{\theta, q}^{1+\eta(2-p) /(p-1)}
$$

\section{A REGULARITY RESULT FOR THE KORTEWEG-DE VRIES EQUATION}

Consider the initial-value problem for the Korteweg-de Vries equation

$$
\left\{\begin{array}{l}
u_{t}+u u_{x}+u_{x x x}=0, \quad x \in \mathbf{R}, \quad t>0, \\
u(x, 0)=f(x), \quad x \in \mathbf{R}
\end{array}\right.
$$

Remark: The Korteweg-de Vries equation can be derived from a system of hyperbolic equations of the form ${ }^{18}$

$$
\frac{\partial U}{\partial t}+A(U) \frac{\partial U}{\partial x}+D^{(3)}\left(\frac{\partial}{\partial t}, \frac{\partial}{\partial x}, U\right) U=0
$$

where $U$ is a $n \times 1$ column vector, $A(U)$ is an $n \times n$ matrix with distinct real eigenvalues, and $D^{(3)}$ is a homogeneous matrix differential operator, by means of the reductive perturbation expansion, i.e., one looks for the influence of weak dispersion and nonlinearity on a single mode of the linearized version of Eq. (6.2) in the nondispersive limit. As examples of continuum-mechanical systems of the form (6.2) we mention a one-dimensional (1D) model for shallow water waves ${ }^{19}$ and hydromagnetic waves. ${ }^{20}$ Thus the Korteweg-de Vries equation can be considered as an evolution equation for weakly nonlinear and dispersive waves, with applications to different continuum-mechanical systems.

Bona and Scott ${ }^{11}$ proved that the problem (6.1) is well posed in all the Sobolev spaces $H^{s}$ for $s \geqslant 2$. In the proof they used a simple extension of the nonlinear interpolation theorem of Tartar ${ }^{17}$ (cf. Remark 1), which is a special case of our Theorem 1.

Theorem 1: If $s \geqslant 2$ and $f \in H^{s}$, then $u \in C\left(0, t_{0} ; H^{s}\right)$ and

$$
\|u\|_{C\left(0, t_{0} ; H^{s}\right)} \leqslant c h_{s, t_{0}}\left(\|f\|_{H[s]}\right)\|f\|_{H^{s}}
$$

where $h_{s, r_{0}}: \mathbf{R}_{+} \rightarrow \mathbf{R}_{+}$is a continuous nondecreasing function. Moreover, the mapping $u_{0} \rightarrow u$ from $H^{s}$ into $C\left(0, t_{0} ; H^{s}\right)$ is continuous.

Proof (cf. also Ref. 1l): By using some earlier results, Bona-Scott ${ }^{11}$ proved the existence of continuous functions $\psi_{m}: \mathbf{R}_{+} \rightarrow \mathbf{R}_{+}, m \geqslant 1$ such that 


$$
\|u\|_{C\left(0, t_{0} ; H^{m}\right)} \leqslant \psi_{m}\left(\|f\|_{\left.H^{m-1}\right)}\|f\|_{H^{m}}, \quad \forall f \in H^{m}\right.
$$

Moreover, for $m>1$ the mapping $T: H^{m} \rightarrow C\left(0, t_{0} ; H^{m}\right)$ defined by $T f=u$ is continuous. We prove that if $m>2$ and $m-1<s<m$, then $T$ maps $H^{m-1}$ into $C\left(0, t_{0} ; L^{2}\right)$ and there exists a continuous, nondecreasing function $\varphi_{m, t_{0}}: \mathbf{R}_{+} \rightarrow \mathbf{R}_{+}$such that

$$
\|T f-T g\|_{C\left(0, t_{0} ; L^{2}\right)} \leqslant \varphi_{m, t_{0}}\left(\|f\|_{H^{m-1},}\|g\|_{H^{m-1}}\right)\|f-g\|_{L^{2}}
$$

For $m>2$, the mapping $T: H^{m-1} \rightarrow C\left(0, t_{0} ; H^{m-1}\right)$ is continuous and therefore also continuous from $H^{m-1}$ to $C\left(0, t_{0} ; L^{2}\right)$. Since $T$ is continuous, it suffices to prove Eq. (6.5) for $f, g$ belonging to some dense subset of $H^{m-1}$. Therefore, suppose $f, g \in H^{\infty}$ and let $w=u-v=T f-T g$. Then $w$ satisfies the initial-value problem

$$
\left\{\begin{array}{l}
w_{t}+\frac{1}{2}[(u+v) w]_{x}+w_{x x x}=0 \\
w(x, 0)=f(x)-g(x)
\end{array}\right.
$$

We get

$$
\begin{aligned}
\frac{d}{d t}\left(\int_{\mathbf{R}} w^{2}(x, t) d x\right) & =-2 \int_{\mathbf{R}} w w_{x x x} d x-\int_{\mathbf{R}} w[(u+v) w]_{x} d x \\
& =2 \int_{\mathbf{R}} w_{x} w_{x x} d x+\int_{\mathbf{R}} w_{x}(u+v) w d x \\
& =\int_{\mathbf{R}}\left(w_{x}^{2}\right)_{x} d x+\frac{1}{2} \int_{\mathbf{R}}(u+v)\left(w^{2}\right)_{x} d x=-\frac{1}{2} \int_{\mathbf{R}}(u+v)_{x} w^{2} d x
\end{aligned}
$$

Then, by using the Sobolev inequality

$$
\frac{d}{d t}\|w(t, \cdot)\|_{L^{2}}^{2} \leqslant \frac{1}{2}\left(\|u\|_{H^{2}}+\|v\|_{H^{2}}\right)\|w(t, \cdot)\|_{L^{2}}^{2} \leqslant\left[\psi_{2}\left(\|f\|_{H^{2}}\right)\|f\|_{H^{2}}+\psi_{2}\left(\|g\|_{H^{2}}\right)\|g\|_{H^{2}}\right]\|w(t, \cdot)\|_{L^{2}}^{2}
$$

and, thus, according to Gronwall's inequality

$$
\|w(t, \cdot)\|_{L^{2}}^{2} \leqslant\|w(0, \cdot)\|_{L^{2}}^{2} \varphi_{2, t}\left(\|f\|_{H^{2},}\|g\|_{H^{2}}\right),
$$

where $\varphi_{2, t}(a, b) \equiv \exp \left(\psi_{2}(a) a t+\psi_{2}(b) b t\right)$. Now, by taking the supremum over $t \in\left[0, t_{0}\right]$, we have

$$
\|T f-T g\|_{C\left(0, t_{0} ; L^{2}\right)} \leqslant \varphi_{2, t_{0}}\left(\|f\|_{H^{2},}\|g\|_{H^{2}}\right)\|f-g\|_{L^{2}}
$$

Hence, by using the interpolation Theorem 1 with

$$
\begin{gathered}
A_{0}=L^{2}, \quad B_{0}=C\left(0, t_{0} ; L^{2}\right), \\
A_{1}=H^{m}, \quad B_{1}=C\left(0, t_{0} ; H^{m}\right), \\
\mu=\frac{m-1}{m}, \quad \theta=\frac{s}{m}, \quad r=p=2, \quad \alpha_{0}=\alpha_{1}=1
\end{gathered}
$$

and the facts 


$$
\left(L^{2}, H^{m}\right)_{\theta, 2}=H^{s}
$$

and

$$
\left(C\left(0, t_{0} ; L^{2}\right), C\left(0, t_{0} ; H^{m}\right)\right)_{\theta, 2} \subset C\left(0, t_{0} ;\left(L^{2}, H^{m}\right)_{\theta, 2}\right)=C\left(0, t_{0} ; H^{s}\right), \quad s=\theta m,
$$

we obtain Eq. (6.3). Moreover, since the pair $\left(L^{2}, H^{m}\right)$ admits a $(\theta, 2)$ approximate identity (see Example 1), it follows from Theorem 1 that $T$ is a continuous mapping from $H^{s}$ to $C\left(0, t_{0} ; H^{s}\right)$ and the proof is complete.

\section{FINAL DISCUSSION AND CONCLUDING REMARKS}

\section{A. Some general open questions}

Roughly speaking, we can say that the existing nonlinear interpolation theory is of the type that an interpolation method for linear operators, e.g., the real method ()$_{\theta, q}$ studied in this article, is also an interpolation method for a class of nonlinear operators. The following questions seem to be of great interest both from the point of view of pure mathematics and of applications:

Consider the subclass $N_{0}$ of nonlinear operators.

(a) Describe the biggest subclass such that the real method ()$_{\theta, q}$ is an interpolation method for $L \cup N_{0}$, where $L$ is the class of linear operators (this class surely contains sublinear and Lipschitz operators, see Ref. 9).

(b) The same question applies to the complex and other methods.

(c) Is it possible to make a new construction of interpolation spaces such that these spaces are interpolation methods for a fixed class of nonlinear operators which cannot be covered by the existing interpolation theory of nonlinear operators (which is clearly modeled upon the classical constructions)?

Remark: If a suitable theory suggested in (c) exists, then we can cover applications for a new class of nonlinear PDEs and thus obtain further possibilities for concrete applications.

\section{B. Sobolev or Sobolev-Besov regularity of solutions of variational inequalities via interpolation}

Several articles are devoted to the Sobolev and Sobolev-Besov regularity of solutions to variational inequalities or the Cauchy problem of PDEs using interpolation of linear or nonlinear operators.

A variational inequality can be formulated as follows: Find $u \in K$ such that

$$
(T u-f, v-u) \geqslant 0, \quad \forall v \in K,
$$

where $K$ is a closed convex subset of a Banach space $X, f$ is a fixed element of the dual space $X^{*}$, and $T: X \rightarrow X^{*}$ is a monotone or pscudomonotone operator. In some cases it is possible to approximate the solutions of variational inequalities with solutions of operator equations and get $a$ priori estimates. Then we can use the interpolation theorem of locally Hölder operators to prove the regularity of the solutions of variational inequalities. For more details see Maligranda, ${ }^{12}$ Lions, ${ }^{13-15}$ Tartar, ${ }^{17}$ Boccardo, ${ }^{21}$ Brezis, ${ }^{22}$ Chipot, ${ }^{23}$ Lions-Magenes, ${ }^{24}$ and Tartar. ${ }^{25}$

\section{Further regularity results for solutions of PDEs via interpolation}

Interpolation techniques were used by many authors in the proof of the regularity of the initial-value problems for PDEs:

(i) For the wave equation 


$$
\left\{\begin{array}{l}
u_{t t}-\Delta u=0, \quad x \in \mathbf{R}^{n} \\
u(x, 0)=g(x) \\
u_{t}(x, 0)=f(x)
\end{array}\right.
$$

it was investigated by Peral ${ }^{26}$ and Perzan. ${ }^{27}$ For the Klein-Gordon equation

$$
\left\{\begin{array}{l}
u_{t t} \Delta_{x} u+u=0, \quad x \in \mathbf{R}^{3} \\
u(x, 0)=0 \\
u_{t}(x, 0)=f(x)
\end{array}\right.
$$

it was done by Marshall-Strauss-Wainger, ${ }^{28}$ and for the nonlinear wave equation

$$
u_{t t}-\Delta_{x} u+\varphi(u)=0
$$

by Lions, ${ }^{13}$ Pecher, ${ }^{29}$ Saut, ${ }^{30}$ and Simon. ${ }^{31}$

(ii) For the generalized heat conduction equation

$$
\left\{\begin{array}{l}
u_{t}=\left(a(x, t) u_{x}\right)_{x} \\
u(x, 0)=f(x)
\end{array}\right.
$$

it was done by $\mathrm{Da}$ Prato-Grisvard. ${ }^{32}$

(iii) For the generalized Burgers equation

$$
\begin{cases}u_{i}+\varphi(u)_{x}=0, & x \in \mathbf{R}, \quad t>0, \\ u(x, 0)=f(x), & x \in \mathbf{R}\end{cases}
$$

it was done by De Vore-Lucier. ${ }^{33}$ [Note that when $\varphi(u)=u^{2}$, we have the Burgers equation.]

(iv) For the nonlinear Schrödinger equation (NSE)

$$
u_{\imath}+\varphi\left(|u|^{2}\right) u-i \Delta u=0
$$

see Abdelouhab, ${ }^{34}$ Ponce-Urbina, ${ }^{35}$ and Reed-Simon. ${ }^{36}$

(v) For the Boltzmann equation, see Gustafsson. ${ }^{37}$ For the Navier-Stokes equations and related problems, see Lions, ${ }^{14}$ Borchers-Miyakawa, ${ }^{38}$ and Kozono-Sohr. ${ }^{39}$

\section{A final conclusion}

We claim that linear and nonlinear interpolation theory have now been developed to a stage where they can be used as very powerful tools for the purpose of predicting the regularity of the solutions of PDEs from their boundaries and/or initial data. We are convinced that many new beautiful applications are waiting behind the door which we tried to open. This is the real scope of this article.

'J. Bergh and J. Löfström, Interpolation Spaces. An Introduction (Springer-Verlag, Berlin, 1976).

${ }^{2} \mathrm{H}$. Triebel, Interpolation Theory, Function Spaces, Differential Operators (VEB Deutscher Verlag der Wissenschaften, Berlin, 1978).

${ }^{3}$ S. G. Krein, Yu. I. Petunin, and E. M. Scmenov, Interpolation of Linear Operators (American Mathematical Society, Providence, 1982).

${ }^{4}$ L. Maligranda, Interpolation of Operators and Applications (1926-1990), 2nd ed. (Luleå University, Luleå, 1993), bibliography therein.

${ }^{5}$ M. Riesz, "Sur les maxima des formes bilinéaries et sur les fonctionelles linéares," Acta Math. 44, 465-497 (1926).

'J. Marcinkiewicz, "Sur l'interpolation d'operateurs," C. R. Acad. Sci. Paris 208, 1272-1273 (1939).

${ }^{7}$ J. Peetre, A Theory of Interpolation of Normed Spaces, Notes de Mat. No. 39 (Rio de Janeiro, 1968).

${ }^{8}$ R. Adams, Sobolev Spaces (Academic, New York, 1976). 
${ }^{9}$ L. Maligranda, "On interpolation of nonlinear operators," Comments Math. Prace Mat. 28, 253-275 (1989).

${ }^{10}$ L. Maligranda, "Interpolation of locally Hölder operators," Studia Math. 78, 289-296 (1984).

${ }^{11} \mathrm{~J}$. Bona and R. Scott, "Solutions of the Korteweg-De Vries equation in fractional order Sobolev spaces," Duke Math. J. 43, 87-99 (1976).

${ }^{12}$ L. Maligranda, "Interpolation of some nonlinear operators in Banach spaces," Ph.D. dissertation, University of A. Mickiewicz, Poznan, 1979.

${ }^{13} \mathrm{~J}$. L. Lions, "Some remarks on variational inequalities," in Proceedings of the International Conference Functional Analysis and Related Topics, Tokyo 1969 (University of Tokyo, Tokyo, 1969), Vol. 1070, pp. 269-282.

${ }^{14} \mathrm{~J}$. L. Lions, "Sur quelques inéquations variationelles d'evolution pour les opérateurs du 2eme ent et l'interpolation nonlinéaire," Inst. Nazionale de Alta Mat. Symp. Mat. 7, 97-128 (1971).

$15 \mathrm{~J}$. L. Lions, "Interpolation linéaire et nonlinéaire et régularité," Inst. Nazionale de Alta Mat. Symp. Mat. 7, 443-458 (1971).

${ }^{16} \mathrm{~J}$. Peetre, "Interpolation of Lipschitz operators and metric spaces," Mathematica 12, 325-334 (1970).

${ }^{17}$ L. Tartar, "Interpolation nonlinéaire et régularité," J. Functional Anal. 9, 469-489 (1972).

${ }^{18} \mathrm{~T}$. Taniuti and C. C. Wei, "Reductive perturbation method in nonlinear wave propagation I," J. Phys. Soc. (Jpn.) 24, 941-946 (1968).

${ }^{19}$ D. J. Korteweg and G. de Vries, "On the change of form of long waves advancing in a rectangular channel, and a new type of long stationary waves," Philos. Mag., 39, 422-443 (1895).

${ }^{20}$ G. S. Gardner and G. K. Morikawa, "Similarity in the Asymptotic Behavior of Collision-free Hydromagnetic Waves and Water Waves," Courant Institute of Mathematical Sciences, New York University, Report No. NYO $9082,1960$.

${ }^{21}$ L. Boccardo, "Regulite $W_{0}^{1, p}(2<p<\infty)$ de la solution d'un probleme unilateral," Ann. Fac. Sci. Toulouse 3, 69-74 (1981).

${ }^{22}$ H. Brezis, "Problèmes unilateraux," J. Math. Pure Appl. 51, 1-168 (1972).

${ }^{23} \mathrm{M}$. Chipot, Variational Inequalities and Flow in Porous media, Applied Mathematical Sciences, Vol. 52 (Springer-Verlag, Berlin, 1984).

${ }^{24}$ J. L. Lions and E. Magenes, Problèmes aux Limites Non Homogènes et Applications. I (Dunod, Paris 1968); English translation (Springer-Verlag, Berlin, 1972).

${ }^{25} \mathrm{~L}$. Tartar, "Interpolation nonlinéaire et applications," Thése, Paris, 1971.

${ }^{26} \mathrm{~J}$. C. Peral, " $L^{p}$ estimates for the wave equation," J. Functional Anal. 36, 114-145 (1980).

${ }^{27}$ A. V. Perzan, " $L_{p}$ estimates of solutions of Cauchy problems for one-dimensional hyperbolic equation," Mat. Issled. 73, 51-54 (1983) (Russian).

${ }^{28}$ B. Marshall, W. Strauss, and S. Wainger, " $L^{p}-L^{q}$ estimates for the Klein-Gordon equation," J. Math. Pure Appl. 59, 417-440 (1980).

${ }^{29}$ II. Pecher, "Ein nichtlinearer interpolazionssats uns seine anwendung auf nichtlineare wellengleichungen," Math. Z. 161, 9-40 (1978).

${ }^{30} \mathrm{~J}$. C. Saut, "Applications de l'interpolation nonlinéaire a des problémes d'evolution nonlinéaires," J. Math. Pures Appl. 54, 27-52 (1975).

${ }^{31}$ J. Simon, "Regularité de la solution d'une équation nonlinéaire dans $\mathbf{R}^{N}$," Lect. Notes Math. 665, 205-227 (1978).

${ }^{32}$ G. DaPrato and P. Grisvard, "Maximal regularity for evolution equations by interpolation and extrapolation," J. Functional Anal. 58, 107-124 (1984).

${ }^{33}$ R. DeVore and B. J. Lucier, "High order regularity for solutions of the invsicid Burgers equation," Lect. Notes Math. 1402, 147-154 (1989).

${ }^{34}$ L. Abdelouhab, "Nonlocal dispersive equations in weighted Sobolev spaces," Differential Integral Eqs. 5, $307-338$ (1992).

${ }^{35} \mathrm{G}$. Ponce and W. Urbina, Schrödinger Equation: An Introduction to Nonlinear evolution Equations (Third Venezuelan School of Mathematics, Merida, 1990), pp. 1-92 (Spanish).

${ }^{36}$ M. Reed and B. Simon, Methods of Modern Mathematical Physics II: Fourier Analysis, Self-Adjointness (Academic, New York, 1975).

${ }^{37}$ T. Gustafsson, " $L^{P}$ estimates for the nonlinear spatially homogeneous Boltzman equation," Arch. Rat. Mech. Anal. 92, 23-57 (1986).

${ }^{38}$ W. Borchers and T. Miyakawa, "Algebraic $L^{2}$ decay for Navier-Stokes flows in exterior domains," Acta. Math. 165, $189-227$ (1990).

${ }^{39} \mathrm{H}$. Kozono and H. Sohr, "Density properties for solenoidal vector fields, with applications to Navier-Stokes equations in exterior domains," J. Math. Soc. (Jpn.) 44, 307-330 (1992). 
Journal of Mathematical Physics is copyrighted by the American Institute of Physics (AIP). Redistribution of journal material is subject to the AIP online journal license and/or AIP copyright. For more information, see http://ojps.aip.org/jmp/jmpcr.jsp Copyright of Journal of Mathematical Physics is the property of American Institute of Physics and its content may not be copied or emailed to multiple sites or posted to a listserv without the copyright holder's express written permission. However, users may print, download, or email articles for individual use. 